\title{
CLIFFORD INDICES OF RIBBONS
}

\author{
DAVID EISENBUD AND MARK GREEN
}

\begin{abstract}
We present a theory of "limit linear series" for rational ribbonsthat is, for schemes that are double structures on $P^{1}$. This allows us to define a "linear series Clifford index" for ribbons. Our main theorem shows that this is the same as the Clifford index of ribbons studied by Eisenbud-Bayer in this same volume. This allows us to prove that the Clifford index is semicontinuous in degenerations from a smooth curve to a ribbon. A result of Fong [1993] then shows that ribbons may be deformed to smooth curves of the same Clifford index. Thus the Canonical Curve Conjecture of Green [1984] would follow, at least for a general smooth curve of each Clifford index, from the corresponding statement for ribbons.
\end{abstract}

\section{INTRODUCTION}

A rational ribbon is a double structure on $\mathbb{P}^{1}$; that is, a scheme $C$ over a field $k$, without embedded points, such that $C_{\text {red }} \cong \mathbb{P}^{1}$ and such that

(1) If $\mathscr{I}$ is the ideal sheaf of $C_{\text {red }}$ in $C$, then $\mathscr{I}^{2}=0$, and

(2) $\mathscr{I}$ is a line bundle on $C_{\text {red }}$.

It is easy to see that the arithmetic genus of $C$ is $g$ iff $\mathscr{I}=\mathscr{O}_{\mathrm{P} 1}(-g-1)$.

Rational ribbons and their canonical embeddings were studied in some detail by Bayer and Eisenbud (this volume), as part of a program for proving the Canonical Curve Conjecture of Green [1984] (see also Eisenbud [1992] for an exposition of some of what is known). The conjecture states that the Clifford index of a smooth curve $D$ is equal to a certain invariant of the minimal free resolution of the homogeneous coordinate ring of the canonical image of $D$, which we will call the resolution Clifford index of $D$. Briefly, if $I$ is the ideal of the canonical image of $D$, the resolution Clifford index may be defined as $g-2-p$, where $p$ is the length of the linear part of the free resolution of $I$.

The Clifford index of a smooth curve $D$ is defined in terms of linear series on $D$ : it is the least number $c$ such that there exists a $g_{d}^{r}$ on $D$ with $r \geq 1$, $d \leq g-1$, and $c=d-2 r$. By contrast, the Clifford index of a ribbon $C$ is defined by Bayer and Eisenbud in terms of the decomposition of the sheaf of Kähler differentials on $C$ restricted to $C_{\text {red }}=\mathbb{P}^{1}$ : they show that one may write

$$
\left.\Omega_{C}\right|_{\text {red }} \cong \mathscr{O}_{\mathrm{P}^{1}}(-a-2) \oplus \mathcal{O}_{\mathrm{P}^{1}}(-b-2) \quad \text { with } a \leq b \text {, }
$$

Received by the editors March 15, 1993 and, in revised form, April 18, 1994.

1991 Mathematics Subject Classification. Primary 13H10, 13D02, 14H45, 14H99; Secondary 13D25, $14 \mathrm{H} 10$.

Both authors are grateful to the NSF-the second through grant number DMS 88-02020 for partial support during the preparation of this work. 
and define

Cliff $C:=a$.

In this paper we will show that-in characteristic 0-this is the same as a Clifford index defined from (suitably generalized) linear series. This will allow us to prove that the Clifford index is semicontinuous in degenerations of a smooth curve to a ribbon. Together with results of Fong [1993] showing that one can smooth any ribbon to a smooth curve of the same Clifford index as the ribbon, and some other known results, this shows that the Canonical Curve Conjecture could be proved for a generic smooth curve of each Clifford index by proving the corresponding statement, Clifford index $=$ Resolution Clifford index, for ribbons (this statement for ribbons was conjectured in Bayer-Eisenbud (this volume)). It would be interesting to know whether the characteristic 0 assumption, which comes essentially from our use of a result of Herzog [1992], could be removed.

The geometric part of the proof is relatively straightforward, allowing for the necessary extension of many known results to the nonreduced case. On the other hand the use of the result of Herzog, by means of our Lemma 1.5, seems to us novel, and presented the main difficulty in finding the proof.

An interesting feature of the situation for ribbons is that, in contrast with the situation for reduced curves, the family of generalized line bundles seems not to form a bounded family, so that it is not so clear how to use them to get a good "compactified Jacobian": the set of generalized line bundles of degree 0 on a ribbon $C$ is the disjoint union, over all positive integers $b$ and effective divisors $\beta$ of degree $b$ in $\mathbb{P}^{1}$, of the sets of (ordinary) line bundles of degree $-b / 2$ on $C$ blown up at $\beta$. If $b \geq$ genus $C$ each such set has just one point; but the set of divisors $\beta$ is still too large to form a good family. It would be interesting to know whether one could perhaps compactify in some way within the family of divisors $\beta$ of degree $\leq$ genus $C$, for example (at least to have a bounded, equidimensional space).

\section{GeNeralized LINE BUNDLES AND LINEAR SERIES ON RIBBONS}

On a smooth complete curve, a linear series is a pair $\Phi=(\mathscr{L}, V)$ with $\mathscr{L}$ a line bundle and $V$ a vector space of sections of $\mathscr{L}$. We say that $\Phi$ has degree $d$ and dimension $r$, or that $\Phi$ is a $g_{d}^{r}$, if degree $\mathscr{L}=d$ and $\operatorname{dim} V=r+1$ (as a vector space). In the case of reduced irreducible (but possibly singular) curves, one may build a theory by allowing rank 1 torsion free sheaves, following D'Souza [1979] and others, or, equivalently, by allowing linear series on blowups of the curve to count as linear series on the curve. It turns out that the case of ribbons follows this pattern. However, the theory of torsion free sheaves over schemes with nilpotents is not so familiar as in the reduced irreducible case.

The theory developed in this section works for a ribbon $C$ with $C_{\text {red }}$ any smooth curve-not just $\mathbb{P}^{1}$ (the definition, given explicitly in Bayer-Eisenbud, is exactly parallel to the one for rational ribbons given above). Throughout, we will maintain the notation $\mathscr{I}$ for the ideal of $C_{\text {red }}$ in $C$.

We begin, following Bayer and Eisenbud, by defining a generalized line bundle on a ribbon $C$ to be a coherent sheaf $\mathscr{L}$ such that

(1) $\mathscr{L}$ is torsion free: that is, for each open affine set $U$ of $C$, every 
nonzerodivisor on $\mathscr{O}_{C}(U)$ is a nonzerodivisor on $\mathscr{L}(U)$, and

(2) $\mathscr{L}$ is generically free of rank 1 on $C$. That is, for some open subset $U$ of $C,\left.\mathscr{L}\right|_{U}$ is a line bundle; or equivalently, $\mathscr{L}$ is a line bundle at the generic point of $C$.

As usual we set

$$
\text { degree } \mathscr{L}:=\chi(\mathscr{L})-\chi\left(\mathscr{O}_{C}\right) \text {. }
$$

This notion of generalized line bundle is the same, in our case, as the general notion of generalized divisor developed (independently and unpublished) by Robin Hartshorne.

The structure of generalized line bundles on ribbons can be described very completely in a manner remarkably parallel to what is done for torsion free sheaves on reduced curves: In brief, every generalized line bundle on a ribbon $C$ is the pushforward of a unique (ordinary) line bundle on a unique blowup of $C$, as we will now show.

First some easy remarks: Let $\mathscr{L}$ be a generalized line bundle on a ribbon $C$, and set

$$
\overline{\mathscr{L}}=\left(\left.\mathscr{L}\right|_{C_{\text {red }}} / \text { torsion }\right),
$$

which is a line bundle on $C_{\text {red }}$. We define $e=e(\mathscr{L})$ to be the degree of this line bundle. Let $\mathscr{K}=\mathscr{K}(\mathscr{L})$ be the kernel of the natural map $\mathscr{L} \rightarrow \overline{\mathscr{L}}$, so that we have an exact sequence

$$
0 \rightarrow \mathscr{K} \rightarrow \mathscr{L} \rightarrow \overline{\mathscr{L}} \rightarrow 0 .
$$

Because $\mathscr{L}$ is torsion free and generically free, $\mathscr{K}$ is again a sheaf on $C_{\text {red }}$, and the natural map $\mathscr{I} \otimes \overline{\mathscr{L}} \rightarrow \mathscr{K}$ is injective. Thus we may write $\mathscr{K} \cong \mathscr{I}(\beta)$, for some effective divisor $\beta \subset C_{\text {red }}$. Let $b=b(\mathscr{L}) \geq 0$ be the degree of $\beta$. With this notation,

$$
\text { degree } \mathscr{L}=2 e+b .
$$

If $\mathscr{L}$ is actually a line bundle then $b=0$, and it is easy to see that the converse holds as well (this also follows at once from Theorem 1.1, below).

We next describe a construction of generalized line bundles. Recall from Bayer-Eisenbud that if $\varphi: C^{\prime} \rightarrow C$ is a morphism of ribbons which induces the identity map $C_{\text {red }}^{\prime} \rightarrow C_{\text {red }}$, then $C^{\prime}$ is obtained from $C$ by blowing up a uniquely determined divisor $\beta \subset C_{\text {red }} \subset C$. The map $\varphi^{*}: \mathscr{O}_{C} \rightarrow \varphi_{*} \mathscr{O}_{C^{\prime}}=\mathscr{O}_{C^{\prime}}$ of sheaves of abelian groups on $\mathbb{P}^{1}$ fits into a diagram

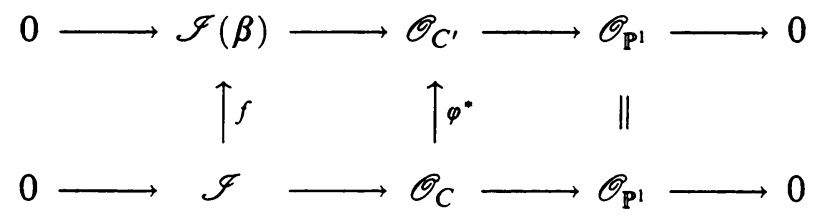

where we have written $f$ for a section of $\mathscr{O}_{\mathrm{Pl}^{1}}(\beta)$ vanishing on $\beta$. If $\mathscr{L}^{\prime}$ is a line bundle on $C^{\prime}$, then $\mathscr{L}:=\varphi_{*} \mathscr{L}$ will be a generalized line bundle on $C$, and with notation as in the remarks above, $\mathscr{K}(\mathscr{L})=\mathscr{I} \otimes \overline{\mathscr{L}}(\beta)$, so that $\mathscr{K}(\mathscr{L}) / \mathscr{L} \mathscr{L}=\mathscr{O}_{\beta}$.

We will show that every generalized line bundle on $C$ arises from a unique $\beta$ and $\mathscr{L}^{\prime}$ in this way: 
Theorem 1.1. Let $\mathscr{L}$ be a generalized line bundle on a ribbon $C$. There is a unique divisor $\beta \subset C_{\text {red }}$ and a unique line bundle $\mathscr{L}^{\prime}$ on the blowup $\varphi: C^{\prime} \rightarrow C$ of $C$ at $\beta$ such that $\mathscr{L} \cong \varphi_{*} \mathscr{L}^{\prime}$.

Remarks. (1) With notation as in the theorem, genus $C^{\prime}=$ genus $C-b$. Since $\chi(\mathscr{L})=\chi\left(\mathscr{L}^{\prime}\right)$, we see that degree $\mathscr{L}^{\prime}=$ degree $\mathscr{L}-b$.

(2) The ribbon $C^{\prime}$ may be written directly as $\operatorname{Spec}(\operatorname{End}(\mathscr{L}))$, but we shall not use this.

Proof. The essential point to prove is that $\mathscr{K}(\mathscr{L}) / \mathscr{I L}$ is generated by one element:

Since $\mathscr{K}(\mathscr{L})$ is a subsheaf of the $\mathscr{O}_{C}$-torsion free sheaf $\mathscr{L}$, it is torsion free. Since it is actually a sheaf of modules on $C_{\text {red }}$, it is a vector bundle on $C_{\text {red }}$. But $\mathscr{L}$ is generically a line bundle on $C$. Thus $\mathscr{K}(\mathscr{L})$ must have rank 1. As the map $\mathscr{I} \otimes \overline{\mathscr{L}} \rightarrow \mathscr{K}(\mathscr{L})$ is an inclusion, and $\mathscr{I} \mathscr{L}$ is its image, we see that $\mathscr{K}(\mathscr{L}) / \mathscr{I L}$ has finite support, and is thus generated by one element, as required.

Because of this we may write $\mathscr{K}(\mathscr{L}) / \mathscr{L} \mathscr{L}$ as $\mathscr{O}_{\beta}$ for some effective divisor $\beta \subset C_{\text {red }}$. Let $f \in H^{0}(\mathscr{O}(\beta))$ be a section vanishing on $\beta$, and let $C^{\prime}$ be the ribbon obtained by blowing up $\beta$ in $C$. We next show that $\mathscr{L}$ is naturally a sheaf of $\mathscr{O}_{C^{\prime}}$-modules. Suppose that $\sigma^{\prime}$ is a section of $\mathscr{O}_{C^{\prime}}$, and that $m$ is a section of $\mathscr{L}$, both defined over an open set $U$ of $C$. Replacing $U$ by a smaller set if necessary, and working entirely over $U$, we may find a section $\sigma$ of $\mathscr{O}_{C}$ with the same image as $\sigma^{\prime}$ in $\mathscr{O}_{C_{\text {red }}}$, and thus there is a section $\tau$ of $\mathscr{I}$ such that $\sigma^{\prime}=\sigma+f^{-1} \tau$. For any $\mathscr{O}_{C^{\prime}}$-module structure on $\mathscr{L}^{\prime}$ we would have to have $\sigma^{\prime} m=\sigma m+\left(f^{-1} \tau\right) m=\sigma m+f^{-1}(\tau m)$, where the right-hand side would be well defined because $\mathscr{L}^{\prime}$ is torsion-free; since $\tau m \in \mathscr{L}$ and $\mathscr{K}(\mathscr{L})=\mathscr{F} \mathscr{L}(\beta)=\mathscr{O}(\beta) \otimes \mathscr{F L}$, this is always well defined, and it is easy to see that it is independent of the choice of $\sigma$, and makes $\mathscr{L}$ into an $\mathscr{O}_{C^{\prime}}$-module as required. Writing $\mathscr{L}^{\prime}$ for $\mathscr{L}$ equipped with this structure, we have of course $\mathscr{L}=\varphi_{*} \mathscr{L}^{\prime}$.

The uniqueness of the blowup $C^{\prime}$-that is, the uniqueness of $\beta$-follows from the computation $\mathscr{K}\left(\varphi_{*} \mathscr{L}^{\prime}\right) / \mathscr{I} \varphi_{*} \mathscr{L}^{\prime}=\mathscr{O}_{\beta}$ made above. The uniqueness of the line bundle $\mathscr{L}^{\prime}$ with $\varphi_{*} \mathscr{L}^{\prime}=\mathscr{L}$ follows because the $\mathscr{O}_{C^{\prime}}$-module structure on $\mathscr{L}^{\prime}$ was derived, using the formula above, from the $\mathscr{O}_{C}$-module structure on $\mathscr{L}$ alone.

We define a generalized linear series (or $g_{d}^{r}$ ) to be a pair $\Phi=(\mathscr{L}, V)$ on $C$ such that $\mathscr{L}$ is a generalized line bundle of degree $d$, and $V \subset H^{0}(\mathscr{L})$ is a vector space of sections which injects into $H^{0}(\overline{\mathscr{L}})$. If $\mathscr{L}$ is actually a line bundle, we will call the generalized linear series an ordinary linear series.

If $\Phi$ is a generalized $g_{d}^{r}$ we define the Clifford index of $\Phi$ to be

$$
\text { Cliff } \Phi=d-2 r
$$

and we define as usual the linear series Clifford index of $C$ to be the minimum over all Clifford indices of generalized $g_{d}^{r}$ 's with $r \geq 1$ and $d \leq g-1$.

Now returning to rational ribbons, we can state the main result of this paper:

Theorem 1.2. If $C$ is a rational ribbon over a field of characteristic 0 , then the Clifford index of $C$ equals the linear series Clifford index of $C$. 
To establish this connection between generalized linear series on $C$ and the Clifford index of $C$, we will need to interpret generalized linear series as some kind of maps to projective spaces. To do this we must be able to remove base loci which may not be Cartier divisors, and we must be able to pass from generalized linear series to ordinary linear series.

Given a generalized linear series $(\mathscr{L}, V)$, with $V \neq 0$, the base point locus is the closed subscheme of $C$ defined by the annihilator of the quotient of $\mathscr{L}$ by the subsheaf generated by $V$. Thus to remove base points, it is natural to replace $\mathscr{L}$ by the subsheaf $\mathscr{L}_{1}$ generated by $V$. The key remark is:

Lemma 1.3. With notation as above, $\mathscr{L}_{1}$ is again torsion free and $\left(\mathscr{L}_{1}, V\right)$ is again a generalized linear series in our sense. Further, $\mathscr{L} / \mathscr{L}_{1}$ has finite support, so degree $\mathscr{L}_{1} \leq$ degree $\mathscr{L}$.

By Theorem 1.1, we may blow up $C$ to get a ribbon $\pi: C^{\prime} \rightarrow C$ and a line bundle $\mathscr{L}^{\prime}$ on $C^{\prime}$ such that $\pi_{*} \mathscr{L}^{\prime}=\mathscr{L}_{1}$. Since $V \subset H^{0} \mathscr{L}^{\prime}=H^{0} \mathscr{L}_{1}$, we get a linear series $\Phi^{\prime}:=\left(\mathscr{L}^{\prime}, V\right)$ on $C^{\prime}$. Since $\mathscr{O}_{C} \subset \pi_{*} \mathscr{O}_{C^{\prime}}$, we see that $V$ generates $\mathscr{L}^{\prime}$ over $\mathscr{O}_{C^{\prime}}$, and thus $\Phi^{\prime}$ is base point free.

Putting these things together, we have proved

Proposition 1.4. If $C$ is a ribbon and $(\mathscr{L}, V)$ is a generalized $g_{d}^{r}$ on $C$ then there is a $b(\mathscr{L})$-fold blowup $C^{\prime}$ of $C$ and a $g_{d^{\prime}}^{r}\left(\mathscr{L}^{\prime}, V^{\prime}\right)$ on $C^{\prime}$ such that

(1) $\mathscr{L}^{\prime}$ is a line bundle, and $\left(\mathscr{L}^{\prime}, V^{\prime}\right)$ is base point free; that is, $\left(\mathscr{L}^{\prime}, V^{\prime}\right)$ is an ordinary base point free $g_{d}^{r}$.

(2) $d^{\prime} \leq d-b$.

Conversely, every such $\left(\mathscr{L}^{\prime}, V^{\prime}\right)$ gives rise to a generalized linear series on $C$, so that Cliff $\mathscr{L}^{\prime} \leq \operatorname{Cliff} \mathscr{L}-b$.

We can now prove our main theorem:

Proof of Theorem 1.2. Write $c^{\prime}$ for the decomposition Clifford index and $c$ for the linear series Clifford index of $C$. As shown in Bayer-Eisenbud, $c^{\prime}$ may be expressed as the minimum number of times it is necessary to blow up $C$ to make it hyperelliptic; that is, any such $C$ has a generalized $g_{c^{\prime}+2}^{1}$. This shows that $c \leq c^{\prime}$, and it remains to prove the opposite inequality: given $\Phi$, a generalized $g_{d}^{r}$ on $C$, with $d \leq g-1$, we must show that $d-2 r \geq c^{\prime}$.

By Proposition 1.4 there is a $b$-fold blowup $C^{\prime}$ of $C$ and an ordinary base point free $g_{d^{\prime}}^{r}$ say $\Phi^{\prime}$, on $C^{\prime}$ with $d^{\prime} \leq d-b$, so

$$
\text { Cliff } \Phi^{\prime} \leq \operatorname{Cliff} \Phi-b \text {. }
$$

By the same result of Bayer-Eisenbud as above, the Clifford index of $C^{\prime}$ is $\geq c^{\prime}-b$. Thus it suffices to prove that Cliff $\Phi^{\prime}$ is $\geq$ the Clifford index of $C^{\prime}$. Further, Bayer-Eisenbud show that $g^{\prime}:=$ genus $C^{\prime}=g-b$, so degree $\Phi \leq g^{\prime}-1$. Changing notation, we may thus assume that $C^{\prime}=C$, $\mathscr{L}$ is a line bundle, and $\Phi$ is base point free to begin with.

Now we switch points of view, and consider $\Phi$ as representing a morphism $\Phi: C \rightarrow \mathbb{P}^{r}$ of degree $d \leq g-1$, such that $\varphi:=\left.\Phi\right|_{C_{\text {red }}}$ has nondegenerate image, and we must show that $d-2 r \geq c^{\prime}$.

Since $\mathscr{L}$ is a line bundle, the degree $d$ of $\mathscr{L}$, which is the degree of $\Phi$, is of the form $d=2 e$, where $e$ is the degree of $\varphi$. We get a commutative 
diagram of cotangent mappings

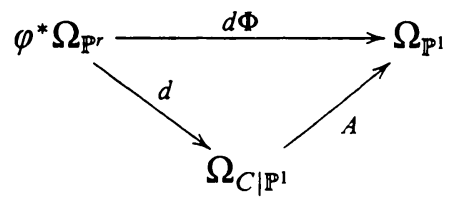

where $A$ is the cotangent mapping of the inclusion $\mathbb{P}^{1}=C_{\text {red }} \subset C$. Since

$$
\Omega_{C \mid \mathbb{P}^{1}} \cong \mathscr{O}_{\mathbb{P}^{1}}\left(-2-a_{1}\right) \oplus \mathscr{O}_{\mathbb{P}^{1}}\left(-2-a_{2}\right) \text { and } \Omega_{\mathbb{P}^{1}} \cong \mathscr{O}_{\mathbb{P}^{1}}(-2) \text {, }
$$

we may regard $A$ as given by a pair of homogeneous polynomials $f_{1}, f_{2}$ of degrees $a_{1}, a_{2}$ respectively.

On the other hand, the map labeled $d \Phi$ may be computed as follows: Pulling back the cotangent sequence

$$
0 \rightarrow \Omega_{\mathbb{P} r} \rightarrow \mathscr{O}_{\mathbb{P r}}(-1)^{r+1} \rightarrow \mathscr{O}_{\mathbb{P} r} \rightarrow 0
$$

on $\mathbb{P}^{r}$ to $\mathbb{P}^{1}$ via $\varphi$, we get an exact sequence on $\mathbb{P}^{1}$

$$
0 \rightarrow \varphi^{*} \Omega_{\mathbb{P} r} \rightarrow \mathscr{O}_{\mathbb{P}^{1}}(-e)^{r+1} \stackrel{\left(\varphi_{0}, \ldots, \varphi_{r}\right)}{\longrightarrow} \mathscr{O}_{\mathbb{P}^{1}} \rightarrow 0,
$$

where $\varphi_{i}$ are the forms defining $\varphi$. We may thus write $\varphi^{*} \Omega_{\mathbb{P}^{r}}$ as the module of syzygies of the $\varphi_{i}$. We set

$$
\varphi^{*} \Omega_{\mathbb{P}} \cong \sum_{i=0}^{r} \mathscr{O}_{\mathbb{P}}\left(-\alpha_{i}-2\right),
$$

where we have called the degrees $-\alpha_{i}-2$ to simplify subsequent notation. The map

$$
d \varphi: \varphi^{*} \Omega_{\mathbb{P} r} \rightarrow \Omega_{\mathbb{P l}^{1}} \cong \mathscr{O}_{\mathbb{P}^{1}}(-2)
$$

is then given by $r$ polynomials $g_{1}, \ldots, g_{r}$, say, with degree $g_{j}=\alpha_{j}$. The commutativity of the diagram above implies that the ideal generated by the $g_{j}$ is contained in the ideal generated by $f_{1}$ and $f_{2}$. If we write $\beta$ for the least total degree of a syzygy among the $g_{j}$, and $b:=\min \left(\beta, a_{2}\right)$, we obtain, from Lemma 1.5 below, the inequality

$$
\sum_{j=1}^{r} \alpha_{j} \geq c^{\prime}+(r-1) b, \quad \text { or } \quad \sum_{j=1}^{r} \alpha_{j}-(r-1) b \geq c^{\prime} .
$$

It thus will suffice to show that

$$
d-2 r=2 e-2 r \geq \sum_{j=1}^{r} \alpha_{j}-(r-1) b .
$$

From the exact sequence defining $\varphi^{*} \Omega_{\mathbb{P}^{r}}$ we get

$$
\sum_{j=1}^{r} \alpha_{j}=(r+1) e-2 r,
$$

so it suffices to show that $b \geq e$. We have $2 e \leq g-1=a_{1}+a_{2}$ by hypothesis, so $e \leq a_{2}$, and we need only show $e \leq \beta$.

We claim, first, that the $g_{j}$ minimally generate the ideal they generate, so that $\beta \geq 1+\min \left(\alpha_{j}\right)$. This follows from Herzog [1994, Corollary 3] where it 
is proved that the $g_{j}$ give minimal generators of the socle of $S /\left(\varphi_{0}, \ldots, \varphi_{r}\right)$, where $S$ is the polynomial ring in two variables, $\sum_{n} H^{0} \mathscr{O}_{\mathbb{P}^{1}}(n)$. (This is the only place in the argument where the assumption of characteristic 0 is used.) Further, since $\varphi$ is by hypothesis nondegenerate, the $\varphi_{i}$ are linearly independent. Thus $\min \left(\alpha_{j}\right)=\min \left(2+\alpha_{j}\right)-2 \geq e+1-2=e-1$. Putting this together we get $\beta \geq e$ as required.

It remains to prove the inequality on degrees of generators used above. More generally, let $\left(f_{1}, \ldots, f_{t}\right) \subset S:=k[x, y]$ be a homogeneous ideal, with

$$
\operatorname{deg} f_{i}=a_{j}, \quad a_{1} \leq \cdots \leq a_{t} .
$$

Suppose $g_{1}, \ldots, g_{r}$ are homogeneous forms, and write $\beta$ for the minimal degree of a syzygy among the $g_{j}$ (where the degree of a syzygy of the form $0=\sum u_{j} g_{j}$ is by definition the "total degree", $\left.\operatorname{deg} u_{j}+\operatorname{deg} g_{j}\right)$.

Lemma 1.5. With notation as above, suppose that $\left(f_{1}, \ldots, f_{t}\right) \supset\left(g_{1}, \ldots, g_{r}\right)$. If we set $b:=\min \left(\beta, a_{2}\right)$, then

$$
\sum_{j=1}^{r} \operatorname{deg} g_{j} \geq a_{1}+(r-1) b .
$$

Proof. We compare the dimensions of $S /\left(f_{1}, \ldots, f_{t}\right)$ and $S /\left(g_{1}, \ldots, g_{r}\right)$ in degree $b-1$. Since $a_{1} \leq b \leq a_{2}$ we get

$$
\operatorname{dim} S /\left(f_{1}, \ldots, f_{t}\right)_{b-1}=a_{1} .
$$

Writing $\{m\}_{+}$for $\max (m, 0)$ we get (because there are no relations on the $g_{j}$ in degrees $\leq b-1)$

$$
\begin{aligned}
\operatorname{dim} & S /\left(g_{1}, \ldots, g_{r}\right)_{b-1}=\operatorname{dim} S_{b-1}-\operatorname{dim}\left(g_{1}, \ldots, g_{r}\right)_{b-1} \\
& =b-\sum_{j=1}^{r}\left\{b-\operatorname{deg} g_{j}\right\}_{+}, \\
& \geq b-\sum_{j=1}^{r}\left(b-\operatorname{deg} g_{j}\right) \\
& =\left(\sum_{j=1}^{r} \operatorname{deg} g_{j}\right)-(r-1) b .
\end{aligned}
$$

The containment of ideals $\left(f_{1}, \ldots, f_{t}\right) \supset\left(g_{1}, \ldots, g_{r}\right)$ now gives us the desired inequality.

\section{LIMITS OF LINEAR SERIES ON RATIONAL RIBBONS}

Let $\pi: S \rightarrow D$ be a flat 1-parameter family of complete smooth genus $g$ curves degenerating to a ribbon: that is, let $D$ be the spectrum of a discrete valuation ring $R$ with closed point 0 and generic point $\eta$, and suppose that $\pi$ is a flat map of finite type such that the geometric generic fiber $C_{\eta}^{-}$is a complete irreducible smooth curve and the special fiber $C_{0}$ is a rational ribbon. We do not impose any further restriction on the singularities of $S$, but it follows from our hypotheses that $S$ is geometrically reduced, irreducible, and locally Gorenstein. 
Suppose that the Clifford index of $C_{\eta}^{-}$is $c$, and let $\Phi_{\eta}^{-}$be a linear series over $C_{\eta}^{-}$having degree $\leq g-1$ and Clifford index $c$. We will construct a generalized linear series $\Phi_{0}$ on the ribbon $C_{0}$ which is the limit of $\Phi_{\eta}$ in a natural sense, and which has Clifford index $\leq c$. As an immediate consequence we obtain the semicontinuity of Clifford index:

Theorem 2.1. The Clifford index of $C_{0}$ (in either of the senses defined in the previous section) is $\leq$ that of $C_{\eta}^{-}$.

The main novelty in the following construction is the use of torsion free sheaves on nonreduced schemes, which necessitates some care.

Proof. After a base change, we may suppose that $\Phi_{\eta}^{-}$is rational over $D$; that is, it comes from a linear series $\Phi_{\eta}$ on $C_{\eta}$. Suppose that $\Phi_{\eta}$ is a $g_{d}^{r}$; that is, it consists of a line bundle $\mathscr{L}_{\eta}$ of degree $d$ and an $r+1$-dimensional vector space $V_{\eta}$ of sections of $\mathscr{L}_{\eta}$. Since the Clifford index of $\Phi_{\eta}$ is minimal among linear series on $C_{\eta}, \Phi_{\eta}$ is base point free. Since the degree of $\Phi_{\eta}$ is so low, it is special. Choosing a section of $\omega_{C_{\eta}} \otimes \mathscr{L}_{\eta}^{-1}$ we may think of $V_{\eta}$ as a subspace of $H^{0} \omega_{C_{\eta}}$. Since $\Phi_{\eta}$ is base point free, we may think of $\mathscr{L}_{\eta}$ as the subsheaf of $\omega_{C_{\eta}}$ generated by $V_{\eta}$.

The line bundle $\omega_{C_{\eta}}$ extends to the line bundle $\omega_{S / D}$ on $S$ and the $R$ module $\pi_{*} \omega_{S / D}$ is free of rank $g$. Choose a free summand $\widetilde{V}$ of rank= $\operatorname{dim}_{K(\eta)} V_{\eta}=r$ extending $V_{\eta} \subset H^{0} \omega_{C_{\eta}}$. Let $\widetilde{\mathscr{L}}$ be the subsheaf of $\omega_{S / D}$ generated by $\widetilde{V}$. Writing $t \in R$ for a generator of the maximal ideal, we set

$$
\begin{gathered}
\mathscr{L}_{0}=\text { image of } \widetilde{\mathscr{L}} \text { in } \omega_{C_{0}}=(\widetilde{\mathscr{L}} / t \tilde{L}) /(\text { torsion }), \\
V_{0}=\tilde{V} / t \tilde{V} \subset \pi_{*} \omega_{S / D} / t \pi_{*} \omega_{S / D}=H^{0} \omega_{C_{0}} .
\end{gathered}
$$

We claim that $\Phi_{0}=\left(\mathscr{L}_{0}, V_{0}\right)$ is a generalized $g_{d^{\prime}}^{r}$ on $C_{0}$ with $d^{\prime} \leq d$, as required.

The fact that $V_{0}$ injects into $\left.H^{0} \mathscr{L}_{0}\right|_{c_{0}}$ follows from the fact that it injects into $H^{0} \omega_{C_{0}}$ together with the following result of Bayer and Eisenbud:

Propostion 2.2. If $C$ is a ribbon of genus $g$, then there is an exact sequence

$$
\left.0 \rightarrow \mathscr{O}_{\mathbb{P}^{1}}(-2) \rightarrow \omega_{C} \rightarrow \omega_{C}\right|_{\mathbb{P}^{1}}=\mathscr{O}_{\mathbb{P}^{1}}(g-1) \rightarrow 0,
$$

so the natural map $\left.H^{0} \omega_{C} \rightarrow H^{0} \omega_{C^{\prime}}\right|_{\mathbf{P}^{1}}$ is an isomorphism.

It remains to see that $\mathscr{L}_{0}$ is a torsion free sheaf in our sense. Since $\mathscr{L}_{0}$ is defined as a subsheaf of $\omega_{C_{0}}$, the only nontrivial part is generic freeness. But because $V_{0}$ has nontrivial projection to $H^{0}\left(\omega_{C_{0}} / t \omega_{C_{0}}\right)$, we see that $\mathscr{L}_{0}$ is generically equal to $\omega_{C_{0}}$, and we are done.

Definition. A family of generalized line bundles is a sheaf $\widetilde{\mathscr{L}}$ on $S$, flat over $D$, whose restriction to any fiber is a generalized line bundle.

Remark. If $\widetilde{\mathscr{L}}$ is a family of generalized line bundles, then $\widetilde{\mathscr{L}}$ is automatically reflexive. But the converse is false: here is an example of a reflexive sheaf $\widetilde{\mathscr{L}}$ on $S$ whose restriction to the generic fiber is a line bundle but whose restriction to the special fiber (though automatically torsion free) is not a generalized line bundle in our sense: 
Example. Consider the family given by

$$
S=\operatorname{Spec} k[x, y, t] /\left(y^{2}-t^{2}\right) \rightarrow D=\operatorname{Spec} k[t],
$$

which might have been gotten, for example by the base change $s=t^{2}$ from the nicer family $\operatorname{Spec} k[x, y, s] /\left(y^{2}-s\right) \rightarrow \operatorname{Spec} k[x]$. Consider the reflexive rank 1 module

$$
M=(y, t) \subset k[x, y, t] /\left(y^{2}-t^{2}\right) .
$$

Being reflexive, $M$ is locally free away from the singular locus $y=t=0$; but restricted to the central fiber, which is the ribbon

$$
\operatorname{Spec} k[x, y] /\left(y^{2}\right) \text {. }
$$

We get

$$
M / t M \cong\{k[x, y] /(y)\} \oplus\{k[x, y] /(y)\} ;
$$

that is, $M / t M$ is killed by $y$, and thus is not generically free on the ribbon.

We can now prove the result that motivated this whole development:

Proposition 2.3. If there is a ribbon of Clifford index $c$ equal to its resolution Clifford index, then a generic smooth curve of Clifford index $c$ satisfies the Canonical Curve Conjecture.

Proof. By Theorem 2.1 and the result of Fong [1993], there is a smoothing $\pi: S \rightarrow D$ with central fiber $C_{0}$ and geometric general fiber $C_{\eta}^{-}$such that the Clifford index of $C_{\eta}^{-}$is $c$ as well. By Green [1984, Appendix] the resolution Clifford index of $C_{\eta}^{-}$is $\leq c$. But by the semicontinuity of free resolutions in families, the resolution Clifford index of $C_{\eta}^{-}$is $\geq$that of $C$, and we are done.

\section{REFERENCES}

D. Bayer and D. Eisenbud, Ribbons and their canonical embeddings, Trans. Amer. Math. Soc. 347 (1995), 719-756.

C. D’Souza, Compactification of generalized Jacobians, Proc. Indian Acad. Sci. Math. Sci. 88 (1979), 419-457.

D. Eisenbud, Green's Conjecture: an orientation for algebraists, Free Resolutions in Commutative Algebra and Algebraic Geometry, Sundance 90, (D. Eisenbud and C. Huneke, eds.), Jones and Bartlett, 1992.

L.-Y. Fong, Rational ribbons and deformations of hyperelliptic curves, J. Algebraic Geom. 2 (1993), 295-307.

M. Green, Koszul cohomology and the geometry of projective varieties, (with an appendix by $\mathbf{M}$. Green and R. Lazarsfeld), J. Differential Geom. 19 (1984), 125-171.

Jürgen Herzog, Canonical Koszul cycles, Aportationes Mat. 6 (1992), 33-41.

Department of Mathematics, Brandeis University, Waltham, Massachusetts 02254

E-mail address: eisenbud@math.brandeis .edu

Department of Mathematics, University of California, Los Angeles, California 90624

E-mail address: mlg@math.ucla.edu 